\title{
Honey promotes angiogeneic activity in the rat aortic ring assay
}

- Objective: To investigate possible effects of honey on angiogenesis, using in vitro analogues of angiogenesis and an endothelial proliferation assay.

- Method: Using an in vitro rat aortic ring assay we compared pseudotubule formation by medicinal honey (Activon), supermarket honey (Rowse) and a honey-based ointment (Mesitran), with that of artificial honey (70\% w/w sugar glucose/fructose). Pseudotubules were analysed using TCS Cellworks AngioSys software. The Angiokit sytem was used to validate the results. Using the MTT [3-(4,5-Dimethylthiazol-2-yl)-2,5-Diphenyltetrazolium. Bromide] assay, toxicity was also assessed on human umbilical vein endothelial cells (HUVEC) directly adherent to plastic.

- Results: All honey preparations stimulated pseudotubule formation, maximal at around $0.2 \%$ honey. Medicinal honeys were more active than Rowse. The effect was not attributable to the sugar content. Among the honeys tested, the Manuka-based Activon preparation reduced residual viable biomass compared with a sugar control at $>0.32 \% \mathrm{v} / \mathrm{v}$ concentration. Rowse had a similar effect only at $2.5 \%$, the highest dose tested.

- Conclusion: The influence of honey constituents on angiogenesis in a wound dressing context is likely to be positive, but would depend on the effective dilution of the honey and the penetration of the active constituents against an osmotic gradient. The extent to which this occurs has yet to be established.

- Conflict of interest: This work was conceived, designed and executed by the authors. Medical honey preparations were supplied unconditionally but free of charge by the distributors.

honey; angiogenesis, in vitro angiogenesis assays; wound healing

K. Rossiter,' BSc, Research Student; A.J. Cooper, ${ }^{1,2}$ MSc PhD, Associate Senior Lecturer;

D. Voegeli, ${ }^{3} \mathrm{PhD}, \mathrm{RN}$, Senior Lecturer; B.A. Lwaleed, ${ }^{2,3} \mathrm{PhD}$, FRCPath, Research Fellow;

I School of Pharmacy and Biomedical Sciences, Portsmouth University, Portsmouth, UK 2 Department of Urology, Southampton University Hospitals NHS Trust, Southampton, UK 3 Faculty of Health Sciences, University of Southampton, Southampton, UK Email: bashir@soton. ac.uk oney has been used in the treatment of wounds in many cultures for centuries, and is even mentioned in the Qur'an (Surah 16. Verse 68, 69, The Bee). Despite its antibacterial and antioxidant properties, its use was superseded by modern dressing and antibiotics. However, due to the emergence of antibiotic-resistant microorganisms and a growing interest in 'natural' medicines, honey has been rediscovered for the treatment of wounds.

The potential effect of topical application of honey on angiogenesis is severely under-investigated. Entering the words 'angiogenesis' and 'honey' together into PubMed yielded only two relevant references. $^{2,3}$ Hepsen et al. described the inhibitory effect of propolis (which is collected by bees and used as a cement in hive construction) on corneal neovascularisation in a rabbit corneal model, ${ }^{2}$ while Molan $^{3}$ generalised that honey '...promotes tissue regeneration through stimulation of angiogenesis and the growth of fibroblasts and epithelial cells...' He cited five references, which are all clinical and observational reports, as opposed to experimental or controlled clinical investigations. Two further references from this review, purporting to show experimental stimulation of angiogenesis by honey, in fact relate to the efficacy of indigenous drugs on infected wounds in buffalo. ${ }^{4,5}$
Given the dearth of phenomenological evidence, we set out to produce some descriptive experiments using laboratory models of angiogenesis, in order to form a basis for future mechanistic studies.

\section{Materials and method \\ Honey types and characteristics}

The honey types used in this study were:

- Artificial - an aqueous solution containing glucose $(35 \% \mathrm{w} / \mathrm{v})$ and fructose $(35 \% \mathrm{w} / \mathrm{v})$. We chose percentage weight per volume, as neat honey is too viscous to measure accurately by volume. Seventy grams of sugars were dissolved to make $100 \mathrm{ml}$ final solution. This recipe conforms broadly with control preparations cited in the literature, which vary slightly in the proportions of sugars used; a typical example of control glucose/fructose solution is given by Osato et al. ${ }^{6}$ This artificial syrup acted as the control against hypertonic effects

- Rowse - a supermarket honey. This was labelled as 'a blend of E.C. (European Community) and nonE.C. honeys'

- Mesitran ointment (Aspen Medical Europe, Redditch) - a formulation containing honey, medical grade hypoallergenic lanolin, sunflower oil, cod liver oil, Aloe barbadensis, vitamins $\mathrm{C}$ and $\mathrm{E}$, and zinc oxide. The honey content is quoted as $47 \%$ - Activon (Theo Manufacturing BV, Maastricht NL 
and Advancis Medical Ltd, Nottingham UK): labelled as 'a medical grade Manuka honey'. No ingredients other than Manuka honey are listed for this product.

\section{Honey dilutions}

Because the viscosity of raw honey makes volumetric dispensing impossible, the initial honey dilution was made to $5 \%$ on a w/v (weight per cent [mass of solute (g) / volume of solution (ml)] x 100)) basis. Put simply, $5 \mathrm{~g}$ honey were used per $100 \mathrm{ml}$ final volume. Thereafter, dilutions were prepared on a more conventional $\mathrm{v} / \mathrm{v}$ (volume per cent [volume of solute (ml) / volume of solution (ml)] x 100)) basis.

For angiogenic assays, a five-fold dilution series was used (to cover a wide range of concentrations between $5-0.008 \% \mathrm{v} / \mathrm{v}$ in 5 steps); for the cytotoxicity experiments, doubling dilutions were effected in situ in accordance with our normal published practice. $^{7}$ All dilutions were made in Medium 200 (an endothelial cell culture medium used for cell maintenance [TCS Cellworks, Buckingham, UK]). All dilutions are expressed as of neat preparation. Mesitran's lower honey content was not compensated for

\section{Cytotoxicity assay}

Preparation methodology is outlined in Table 1 . In terms of the products used:

- All reagents were purchased from Sigma Aldrich, unless otherwise stated

- TCS Cellworks provided the human umbilical vein endothelial cells (HUVEC from pooled donor) - EDTA solution was provided by Sigma Aldrich, Poole, Dorset, UK

- The spectrophotometric plate reader was a Bio-Tek Powerwave 340

- Nunc 96-well adherent-cell culture-grade plates were used.

Results are expressed as toxicity (\% inhibition against control growth, or 100 minus \% residual viable biomass (RVB))

\section{Table I. Preparation methodology for the cytotoxicity and angiogenesis assays.}

\section{Cytotoxicity assay}

Human umbilical vein endothelial cells were maintained adherent to plastic in Medium 200 (TCS Cellworks) and passaged using single strength trypsinethylenediaminetetraacetic acid (EDTA) solution

Cells were seeded into 96 -well adherent cell culturegrade plates $\left(5 \times 10^{3} /\right.$ well) and left to settle for 24 hours

Well columns were filled with $100 \mu \mathrm{l}$ of honey solutions. Columns II and 12 were used for $100 \%$ growth and blank controls (filled only with Medium 200) respectively

Plates were incubated for I hour at $37^{\circ} \mathrm{C}$ and $5 \% \mathrm{CO}_{2}$ washed with PBS and re-filled with I50 $\mu$ l of fresh culture medium

After incubation for a further 48 hours, $50 \mu \mathrm{l}$ of $\mathrm{Img} / \mathrm{ml}$ MTT' $^{8}$ were added for two hours' incubation. The supernatant was discarded and the blue formazan product made within viable cells was solubilised with the addition of a $150 \mu l$ of dimethyl sulphoxide (DMSO) per well

The plate was shaken and the developed colour read by absorbance at $570 \mathrm{~nm}$ in a standard spectrophotometric plate reader (Bio-Tek Powerwave 340)

\section{Angiogenesis assays}

Frozen matrigel was equilibrated to its liquid state overnight on ice, and maintained until needed. $200 \mu$ l was transferred into the central 24 wells of a 48 -well plate on ice. The outer wells were filled with saline to act as a barrier to evaporation artefacts and do not feature in analysis. The plate was left in a cold room or fridge to allow it to set

Lengths of rat aorta were stripped of fat and connective tissue, sprayed with $70 \%$ alcohol and immediately immersed in $>20 \mathrm{ml}$ of saline. One millimetre-thick rings were cut and a single ring transferred to each of the matrigel coated wells

$200 \mu \mathrm{l}$ of Medium 200 were added to each test well and surrounding wells were half-filled with phosphate buffered saline (PBS) to minimise water loss

After 2-3 days in the cell culture incubator, $200 \mu$ l of test media were added when initial signs of outgrowth indicated a healthy explant. Honey dilutions were adjusted to account for the $200 \mu$ l of medium already in the wells, but not the volume of gel under the cells

After a further 3-4 days, the outgrowths were ready for analysis. All plates were fixed with an excess of $5 \%$ formalin, which was washed extensively with water and stained with $0.5 \mathrm{ml} 0.01 \%$ methylene blue

Adequate staining was achieved in 30 minutes, and the plates were washed and air dried. Explants and their outgrowths were photographed under a low power stereo-zoom microscope (at $\sim 40 x$ overall magnification), allowing the entire well to be imaged 


\section{Angiogenesis assays}

- Aortic ring assay Again, the methodology is outlined in Table 1. Matrigel is an artificial basement membrane material of murine origin that is used as a substrate on which endothelial cells can differentiate and form tubular structures under appropriate (angiogenic) stimulation. For this study, frozen Matrigel (Becton-Coulter UK) was used.

In a separate experiment, VEGF dilutions were used in order to demonstrate that a known angiogenic stimulator was effective in the assay.

Analysis was as for the Angiokit multi-well in vitro assay system (below) using the entire area of the outgrowth and using the same manufacturer's AngioSys software (TCS Cellworks) for analysis.

- TCS Cellworks Angiokit This assay kit comprises multi-well plates with endothelial cells (probably fibroblasts of murine origin, the kit is patent protected) pre-seeded in a feeder layer, and a given laboratory protocol (see TCS Cellworks website for details). Following exposure to the honeys or control syrup at the concentrations given for each experiment and incubation for one week, the plate was fixed with ice cold $70 \%$ ethanol and the wells were stained immunochemically (e.g. for von Willebrand factor, which identifies endothelial cells from the feeder layer cells of mesodermal origin). After staining, the wells were photographed and analysed using AngioSys software (TCS Cellworks), which provides a number of measures of pseudotubule density length and branching.

The method is too expensive to use routinely, but one plate was also run to assess the software's ability to analyse images of aortic rings stained with methylene blue in the previous angiogensis assay involving the frozen matrigel. Fig 1 illustrates the patterns of pseudotubule formation obtained using each system. Results were found to be comparable when checked against semi-subjective assessments - primarily manual counting of tubule intersection density on a jpeg image of the original preparation, estimating the area of outgrowth by projection onto paper, then cutting out and weighing this area.

\section{Statistical analysis}

Cytotoxicity data was obtained from eight replicates that were identified as normally distributed, with median values that were the same as arithmetic means. The experiment was duplicated, but Fig 2 illustrates the means and standard errors from just a single run, as MTT colour development varied between experiments.

Aortic ring assays were performed with duplicate wells and averaged. Complete experiments were performed three times. The number of wells per treatment was therefore small, however, curve-fitting analysis (direct from the Excel spreadsheet) was applied so that the $\mathrm{R}^{2}$ value obtained reflected the
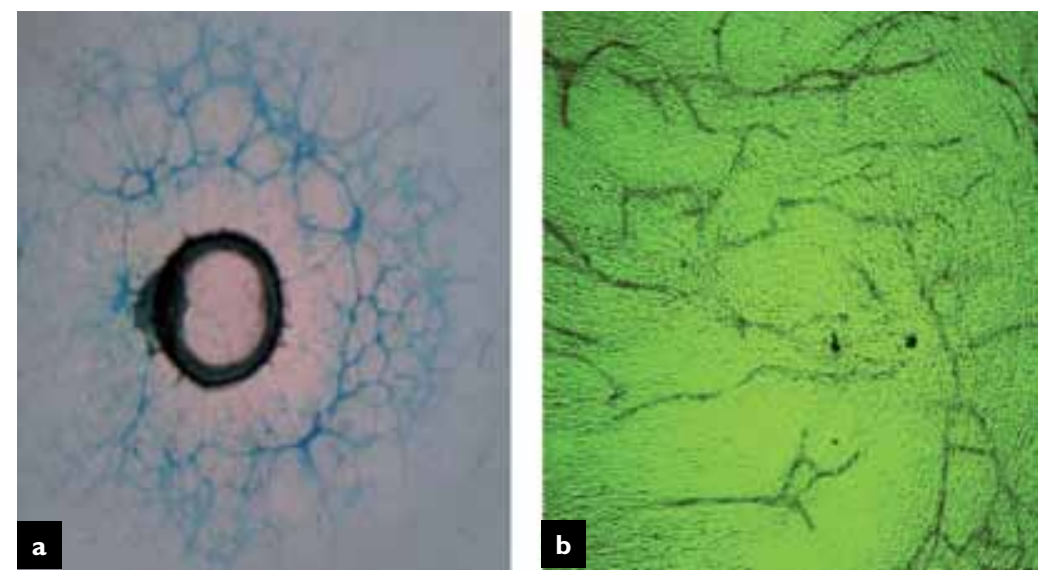

Fig I. Pseudotubule formation in the aortic ring and Angiokit assays: well with aortic ring stained with methylene blue (a); well of Angiokit stained with DAB (3.3'-diaminobenzidine) serving as chromogenic substrate for peroxidaseconjugated antibodies against von Willebrand factor

Fig 2. Cytotoxicity of honey preparations on monolayers of HUVEC cells on plastic

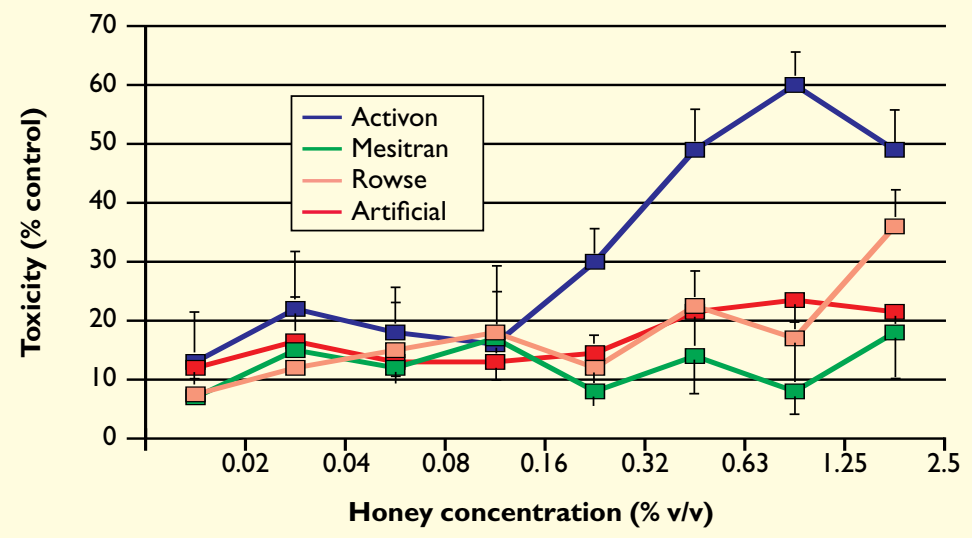

Results are plotted as the means and SEM of eight replicate wells. Toxicity is calculated as 100 minus the \% residual viable biomass relative to untreated control wells

consistency of all data points in conforming to a mathematically defined model, in this case a polynomial equation - i.e., one with known variables, traditionally solved in mathematics by progressively eliminating them until only the 'root' remains.

\section{Results \\ Cytotoxicity}

Only Activon showed a marked cytotoxic dose response over the range studied, with toxicity first rising at $0.32 \% \mathrm{v} / \mathrm{v}$ (Fig 2). Rowse honey only became marginally more toxic at $2.5 \% \mathrm{v} / \mathrm{v}$, the highest dose given; the other two preparations were non-toxic at all doses. The standard errors are such that there are no statistically significant pair-wise differences between the three highest dose readings for Activon $\left(R^{2}=0.8275\right)$. 
Fig 3. Rat aortic ring assay results

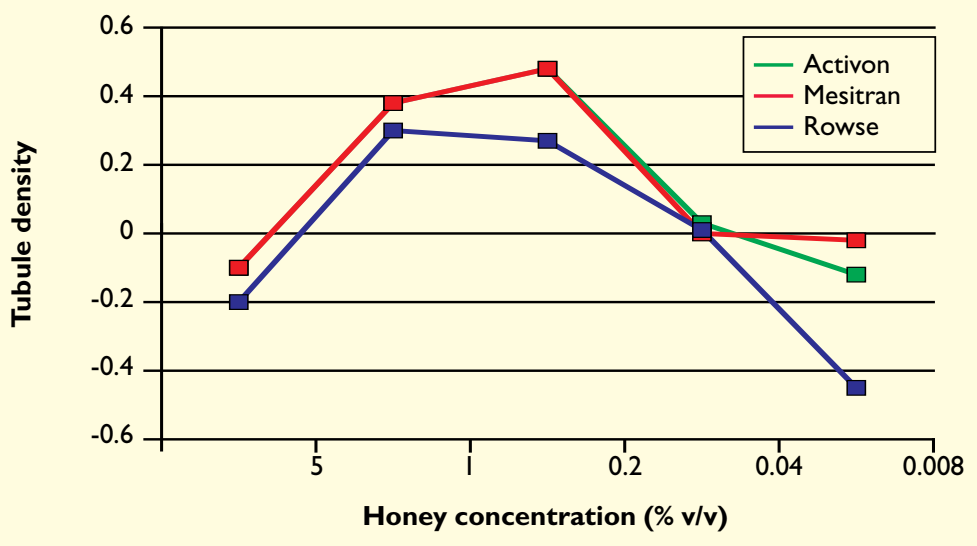

Mean values for tubule density are plotted against serial dilutions of honeys, corrected by subtraction for the relevant artificial honey (sugar) controls. $\mathrm{R}^{2}$ value for polynomial curve-fit: Rowse 0.982 , Mesitran 0.843 , Activon 0.680

Fig 4. Rat aortic ring assay results

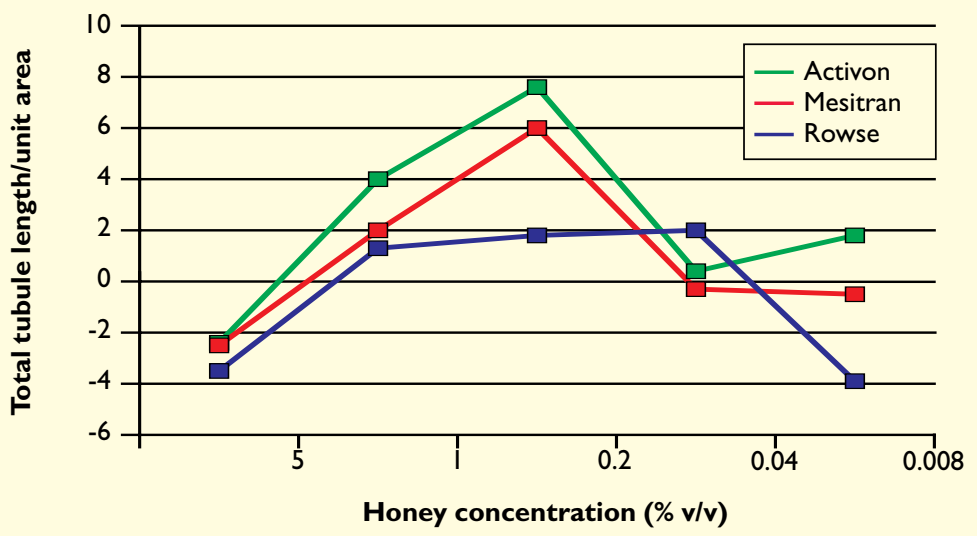

Mean values for total tubule length per unit area are plotted against serial dilutions of honeys, corrected by subtraction for the relevant artificial honey (sugar) controls. $R^{2}$ values for polynomial curve-fit: Rowse 0.942 , Mesitran $0.0 .68 \mathrm{I}$, Activon 0.573 . the honey values. Results for all products tested were similar, showing a bell-shaped response curve, with the highest pro-angiogenic effect found at $0.2 \% \mathrm{v} / \mathrm{v}$ honey. Comparable results were obtained with the other measures available from the software (node density and tubule area).

In a separate experiment, a wide range of VEGF dilutions (in tenfold steps from 200ng/ml) and a range of Activon dilutions, known to cover peak pseudotubule stimulation, were run on the same plate. Tubule-formation curves are shown in Fig 5. Activon was chosen for this purpose as it was the most active product in the other assays and purified VEGF is expensive. The $\mathrm{x}$-axis has been left unlabelled as the two sets of dilutions are not directly comparable, although they cover the concentrations of each agent, giving an in vitro pro-angiogenic response. These responses, measured on the y-axis, are comparable and show the honey to be as strong a stimulator of pseudotubule formation as VEGF in the aortic ring assay. It should be noted that aortic outgrowth is expressed relative to artificial honey data.

- TCS Cellworks Angiokit In the three plates used, honeys at dilutions of $0.2 \%$ and $0.04 \%$ were found to be pro-angiogenic. At 5\% they were antiangiogenic, and at $1 \%$ neutral in their effect.

\section{Discussion}

Wound healing is a complex sequence of coordinated events modified by delicately orchestrated pathways of cytokines and growth factors, that restore the structural integrity of damaged tissues. ${ }^{9}$ Honey has wound-healing properties, has no adverse effects on wound tissues and is cost-effective. ${ }^{10,11,12}$ The antimicrobial and anti-inflammatory properties of honey initiate healing in recalcitrant wounds ${ }^{13}$ by:

- Hastening sloughing of devitalised tissue

- Attracting macrophages to the wound bed

- Providing a local cellular energy source

- Counteracting malodour

- Promoting granulation and epithelialisation

- Protecting the wound from infection. ${ }^{14}$

Honey reduces inflammation at a molecular level, even in the absence of infection, which suggests that its anti-inflammatory effect is direct rather then a secondary consequence to clearing infection via its antibacterial activity. ${ }^{15}$ In vitro, it stimulates cultured monocytes to release cytokines, ${ }^{16}$ and activates the immune system by stimulating mitogenesis in $\mathrm{B}$ and $\mathrm{T}$ lymphocytes and activating neutrophils. ${ }^{17}$ It has been shown to stimulate the production of antibodies in mice exposed to Escherichia coli antigens. ${ }^{18}$ Several in vivo and in vitro studies have demonstrated its direct anti-inflammatory activity ${ }^{19-21}$ and ability to decrease oxidative stress, by mopping up free radicals arising from 
burns. $^{22}$ Together, these studies indicate that honey possesses a broad-spectrum of activity that can positively impact on wound healing at a cellular level in in vitro models. However, other mechanisms involved in the promotion of rapid healing remain unidentified. It is unknown whether or not one therapeutic agent or a single process can ameliorate all issues of repair, or cover all vulnerabilities of impaired wound healing. ${ }^{9}$

Although scant, the available evidence shows honey to be pro-angiogenic when applied to animal wounds. $^{4,5}$ This may account for the clinically observed rapid development of granulation tissue, ${ }^{23,24}$ as granules are fibroblasts growing at sites where there are capillary bundles supplying oxygen. The mechanism(s) by which honey might stimulate angiogenesis are unknown ${ }^{3}$ and with so little work published describing a pro-angiogenic effect, it would be premature to speculate extensively about modes of action at this time.

Topical application of hydrogen peroxide enhances cutaneous blood recruitment in ischaemic ulcers ${ }^{25}$ and stimulates the proliferation of fibroblasts. ${ }^{26}$ As honey is diluted by wound exudate, it produces hydrogen peroxide by glucose oxidase. Honey also induces production of interleukin-6 (IL-6), interleukin-1 $\beta$ (IL-1 $\beta$ ) and tumour necrosis factoralpha (TNF- $\alpha$ ) in monocytes. ${ }^{16}$ The latter is a wellestablished stimulator of angiogenesis. ${ }^{27}$ IL-6 is known to have a significant impact on wound healing $^{16}$ and TNF- $\alpha$ exerts much of its beneficial effect by causing an increase in levels of IL- $6{ }^{28}$ A synergistic effect between these two cytokines has been indicated. ${ }^{29}$ Both TNF- $\alpha$ and IL- $1 \beta$ stimulate the release of various growth factors, including plateletderived growth factor and transforming growth factor-beta. ${ }^{30}$

In this phenomenological study, we investigated the effect of three honey preparations and a sugar syrup (control) on angiogenesis using a rat aortic ring assay, validated by a TCS Cellworks Angiokit assay. Overall, our results suggest that honey is proangiogenic at concentrations of $0.1-1.0 \% \mathrm{v} / \mathrm{v}$. Despite the limited replication of experiments (due to the nature of the assay), the consistency of results across all three honey products lends weight to these results - all were pro-angiogenic within the same dose range, although the greatest effect was observed for Activon.

Dose responses to honey were best described by polynomial equations. The better fit was given by tubule density (Fig 3) where both Rowse and Mesitran gave $R^{2}$ values $>0.8$. Only Rowse gave a good curve-fit $\left(\mathrm{R}^{2}=0.942\right)$ when tubule length was the criterion (Fig 4). Despite Rowse giving the best fit to a polynomial curve, it gave the least elevation of tubule formation; Activon yielded the highest peak.

Clinically, the minimum inhibitory concentra- tion of honey to prevent bacterial growth is $2 \% .{ }^{15}$ The Manuka-based Activon preparation was cytotoxic at this concentration when applied to monocultures of HUVEC that were directly adherent to plastic. Since the concentration of hydrogen peroxide generated from diluted honey is very low compared with that typically applied to a wound, any cytotoxic damage due to hydrogen peroxide would be minimal. ${ }^{31}$ The model described here is likely more sensitive in terms of cytotoxicity than an explant-based culture involving cooperating cell types, ${ }^{32}$ which is an option for the future. This also probably applies to the feeder-layer method represented by the Angiokit system.

The level of response that can be regarded as proangiogenic was comparable to that achieved with a classical stimulator of angiogenesis, VEGF. This contrasts with the reported insensitivity of the HUVECon-matrigel assay to $\mathrm{VEGF},{ }^{33}$ and is the main reason why rat aortic rings are, counter-intuitively, often preferred as an in vitro correlate of angiogenesis. ${ }^{34}$ Although specialist medical grade honey preparations are generally more effective than blended commercial honeys, all produced a similar response curve in the rat aortic ring assay.

Fig 2 shows Activon to be toxic at higher concentrations. The apparent dip in toxicity at the highest concentration is not statistically significant, and is probably due to increased variance of optical densities when measuring low residual biomass.

Interestingly, the apparent pro-angiogenic effect of honey does not depend on sugar content. Indeed, artificial sugar syrup had no significant effect on the assays employed here. In other studies, sugar has been found to be less effective than honey in both reducing bacterial contamination and promoting

Fig 5. Comparison of pseudotubule formation curves by Activon honey and VEGF. Total tubule length is plotted on the $y$-axis

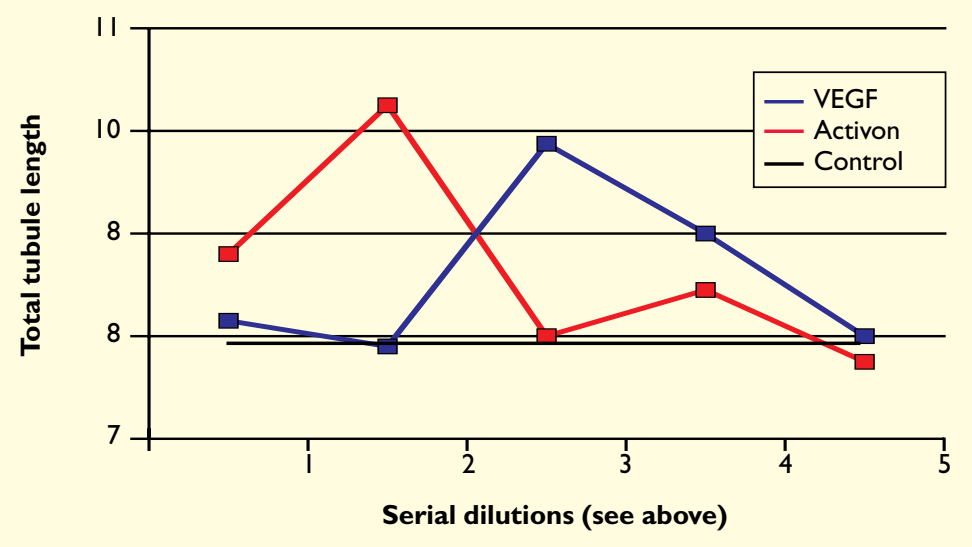

The $\mathrm{x}$-axis is logarithmic, the serial dilutions for VEGF are tenfold, from $\log _{10} 4.3$ to $0.3 \mathrm{pg} / \mathrm{ml}$ and for Activon 0.7 to $-2.8 \% \mathrm{v} / \mathrm{v}$ in fivefold steps. Both curves show a peak $(2 \mathrm{ng} / \mathrm{ml}$ for VEGF and $1 \% \mathrm{v} / \mathrm{v}$ for Activon) with inhibition at higher concentrations. The control in this experiment was a medium blank, as indicated by the horizontal line 
wound healing. ${ }^{35,36}$ Sugar solution does not inhibit bacterial growth in vitro when compared with Manuka honey, which is effective at concentrations of just $5 \% .^{35}$ Furthermore, sugar solution by itself does not cause significant expression of cytokines in monocytes, whereas honey does stimulate them. ${ }^{16}$ This suggests that the regulatory effects of honey could be due to components other than sugars. Honey has antimicrobial activities beyond the osmotic effect of its sugar content, ${ }^{3}$ but in a wound dressing context, the osmotic effect of sugary honey acting as a poultice and causing a net flow of solvent towards the wound surface means that any penetration of minor constituents into the wound bed would have to be against the fluid flow.

\section{References}

I Green,A.E.Wound healing properties of honey. Br J Surg. 1988; 75: 1278.

2 Hepsen, I.F., Er, H., Cekiç, O. Topically applied water extract of propolis to suppress corneal neovascularization in rabbits. Ophthalmic Res. 1999; $31: 6$, 426-43I.

3 Molan, P.C. Potential of honey in the treatment of wounds and burns. Am J Clin Dermatol. 200I; 2: I, 13-19.

4 Gupta, S.K., Singh, H., Varshney, A.C., Prakash, P.Therapeutic efficacy of honey in infected wounds in buffaloes. Indian J Anim Sci. 1992; 62: 6, 521-523.

5 Kumar,A., Sharma,V.K., Singh, H.P. et al. Efficacy of some indigenous drugs in tissue repair in buffaloes. Indian Vet J. 1993; 70 I, 42-44.

6 Osato, M.S., Reddy, S.G. Graham, D.Y. Osmotic effect of honey on growth and viability of Helicobacter pylori. Dig Dis Sci.

1999; 44: 3, 462-464.

7 Jennings, A.M., Solomon, L.Z., Sharpe, P. et al. Estramustine reversal of resistance to intravesical epirubicin chemotherapy. Eur Urol 1999; 35 : 4, 327-335.

8 Osuagwu, F.C., Oladejo, O.W. Imosemi, I.O. et al. Enhanced wound contraction in fresh wounds dressed with honey in

Wistar rats (Rattus Novergicus)

West Afr J Med. 2004; 23: 2 ,

II4-II8.

9 Dart,A.J., Dowling, B.A., Smith, C.L. Topical treatments in equine wound management. Vet Clin

North Am Equine Pract. 2005; 21 : I, 77-89.

10 Ndayisaba, G., Bazira, L., Habonimana, E., Muteganya, D. [Clinical and bacteriological outcome of wounds treated with honey. An analysis of a series of 40 cases] [Article in French] Rev
Chir Orthop Reparatrice Appar Mot. 1993; 79: 2, III-II 3.

I I Moghazy,A.M., Shams, M.E., Adly, O.A. et al. The clinical and cost effectiveness of bee honey dressing in the treatment of diabetic foot ulcers. Diabetes Res Clin Pract. 2010; 89: 3, 276-28I.

12 Eddy, J.J., Gideonsen, M.D. Mack, G.P. Practical considerations of using topical honey for neuropathic diabetic foot ulcers: review.WMJ. 2008; 107: 4, 187-190.

13 Molan, P.C. Re-introducing honey in the management of wounds and ulcers - theory and practice. Ostomy Wound Manage. 2002; 48: II, 28-40.

I 4 Staunton, C.J., Halliday, L.C., Garcia, K.D.The use of honey as a topical dressing to treat a large, devitalized wound in a stumptail macaque (Macaca arctoides). Contemp Top Lab Anim Sci. 2005 ; 44: 4, 43-45.

15 Molan, P.C. The evidence supporting the use of honey as a wound dressing. Int J Low Extrem Wounds. 2006; 5: I, 40-54.

16 Tonks, A., Cooper, R.A., Price, A.J. et al. Stimulation of TNF-alpha release in monocytes by honey. Cytokine. 200I; I4: 4, 240-242. 17 Abuharfeil, N.,Al-Oran, R, Abo-Shehada M.The effect of bee honey on the proliferative activity of phagocytes. Food Argic Immunol. 1999; II: I69-177.

I 8 Al-Waili, N.S., Haq,A. Effect of honey on antibody production against thymus-dependent and thymus-independent antigens in primary and secondary immune responses. J Med Food. 2004; 7: 4 49|-494.

19 Church, J. Honey as a source of the anti-stiffness factor.

Federation Proc Am Physiol Soc. 1954; I3: I, 26.

20 Bilsel,Y., Bugra, D., Yamaner, S. et al. Could honey have a place in

\section{Conclusion}

Honey's most attractive medicinal feature is perhaps its antimicrobial activity, which is likely free from bacterial resistance. ${ }^{37}$ However, other properties of honey are also important, at least in terms of the impact they may have on wound management. We believe that this study increases knowledge of the range of effects that honey can exert in a wound healing context, through indicating a stimulatory effect on angiogenesis. It seems there are possibilities for both positive and negative effects on angiogenesis, largely depending on the depth of penetration by its active constituents against an osmotic gradient. The extent to which this occurs deserves further investigation.

colitis therapy? Effects of honey, prednisolone, and disulfiram on inflammation, nitric oxide, and free radical formation. Dig Surg. 2002; 19: 4, 306-312.

2 I Aysan, E.,Ayar, E., Aren, A Cifter, C. The role of intraperitoneal honey administration in preventing post-operative peritoneal adhesions. Eur J Obstet Gynecol Reprod Biol. 2002; 104: 2 , 152-155.

22 Subrahmanyam, M., Shahapure, A.G., Nagane, N.S. et al. Free radical control - the main mechanism of the action of honey in burns. Ann Burns Fire Disasters. 2003; 16: I35-138.

23 Subrahmanyam, M.A prospective randomised clinical and histological study of superficial burn wound healing with honey and silver sulfadiazine. Burns. 1998; 24: 2, |57-|6|.

24 Efem, S.E. Recent advances in the management of Fournier's gangrene: preliminary observations. Surgery. 1993; I I 3: 2, 200-204.

25 Tur, E., Bolton, L., Constantine, B.E.Topical hydrogen peroxide treatment of ischemic ulcers in the guinea pig: blood recruitment in multiple skin sites. J Am Acad Dermatol. 1995; 33:2 Pt I. 217-221.

26 Chung, L.Y., Schmidt, R.J., Andrews, A.M.,Turner, T.D. A study of hydrogen peroxide generation by, and antioxidant activity of, Granuflex (DuoDERM)

Hydrocolloid Granules and some other hydrogel/hydrocolloid wound management materials. $\mathrm{Br}$ J Dermatol. 1993; I29: 2, |45-153.

27 Leibovich, S.J., Polverini, P.J., Shepard, H.M. et al. Macrophageinduced angiogenesis is mediated by tumour necrosis factor-alpha. Nature. 1987; 329: 61 40, 630-632. 28 Kohase, M., HenriksenDeStefano, D., May, L.T. et al. Induction of beta 2 -interferon by tumor necrosis factor: a homeostatic mechanism in the control of cell proliferation. Cell. 1986; 45: 5, 659-666.

29 Tang, A., Gilchrest, B.A.

Regulation of keratinocyte growth factor gene expression in human skin fibroblasts. J

Dermatol Sci. 1996; I I: I, 4I-50. 30 Slavin, J.The role of cytokines in wound healing.J Pathol. 1996; I78: I, 5-10.

3 I Bang, L.M., Buntting, C., Molan, P.The effect of dilution on the rate of hydrogen peroxide production in honey and its implications for wound healing. I

Altern Complement Med. 2003; 9 : 2, 267-273.

32 Crook, T.J., Dyer, J.P.,

McCormick, R.I. et al. The effects of meglumine gamma linolenic acid (MeGLA) on an organ culture model of superficia bladder cancer. Urol Res. 2002; 30 : I, 59-65.

33 Auerbach, R., Lewis, R., Shinners, B. et al. Angiogenesis assays: a critical overview. Clin Chem. 2003; 49: I, 32-40.

34 Go, R.S., Owen,W.G. The rat aortic ring assay for in vitro study of angiogenesis. Methods Mol Med. 2003; 85: 59-64.

35 al Somal, N., Coley, K.E., Molan, P.C., Hancock, B.M. Susceptibility of Helicobacter pylori to the antibacterial activity of manuka honey. J R Soc Med. 1994; 87: I, 9-12.

36 Mphande,A.N., Killowe, C., Phalira, S. et al. Effects of honey and sugar dressings on wound healing.J Wound Care. 2007; I6: 7, 317-319.

37 Glasser, J.S., Guymon, C.H., Mende, K. et al.Activity of topical antimicrobial agents against multidrug-resistant bacteria recovered from burn patients. Burns. 2010 Jun 7. [Epub ahead of print] 